\title{
Neutron imaging methods for the investigation of energy related materials
}

\section{Fuel cells, battery, hydrogen storage and nuclear fuel}

Eberhard H. Lehmann ${ }^{\text {a }}$, Pierre Boillat, Anders Kaestner, Peter Vontobel and David Mannes

Paul Scherrer Institut, 5232 Villigen PSI, Switzerland

\begin{abstract}
After a short explanation of the state-of-the-art in the field of neutron imaging we give some examples how energy related materials can be studied successfully. These are in particular fuel cell studies, battery research approaches, the storage of hydrogen, but also some investigations with nuclear fuel components. The high contrast for light isotopes like $\mathrm{H}-1$, Li-6 or B-10 are used to trace low amounts of material even within compact sealing of metals which are relatively transparent for neutrons at the same time.
\end{abstract}

\section{Introduction}

Neutrons have the ability to penetrate materials samples and enable in this manner to perform investigations in a non-destructive or non-invasive way. Similarly to X-rays, the analysis of the transmitted radiation can be used in comparison to the initial beam intensity, and information about content and thickness of the investigated material can be derived.

While X-rays are very common in medical inspections in hospitals and for failure analysis in industry, neutron imaging facilities are generally rare and neutron imaging studies are less common. This situation is caused by the low number of reasonably intense neutron sources and the limited access to suitable beam ports there. Nowadays, modern neutron imaging systems can provide the same image quality than X-ray facilities, however the inherent contrast mechanism and the derived image information differs systematically as shown in Fig. 1 as an example. The reasons will be explained below.

With respect to investigations for energy related materials it was quite common and important some years ago to study nuclear fuel elements with neutrons since uranium is very absorbing for X-rays while thermal neutrons can transmit $10 \mathrm{~mm}$ of natural uranium quite easily. As the isotope U-235 has a much higher attenuation coefficient than U-238 it is possible to derive the degree of the enrichment of the fuel from neutron radiography data directly and with good accuracy non-invasively (see below).

As nuclear energy should have politically less importance in the future, the research today is more focused onto alternative energy supply, including energy storage and conversion. In this context, neutron imaging methods can also be applied and used favorably. This article is concentrated mostly on

\footnotetext{
${ }^{\text {a }}$ Corresponding author: eberhard.lehmann@psi.ch
}

This is an Open Access article distributed under the terms of the Creative Commons Attribution License 4.0, which permits unrestricted use, distribution, and reproduction in any medium, provided the original work is properly cited. 


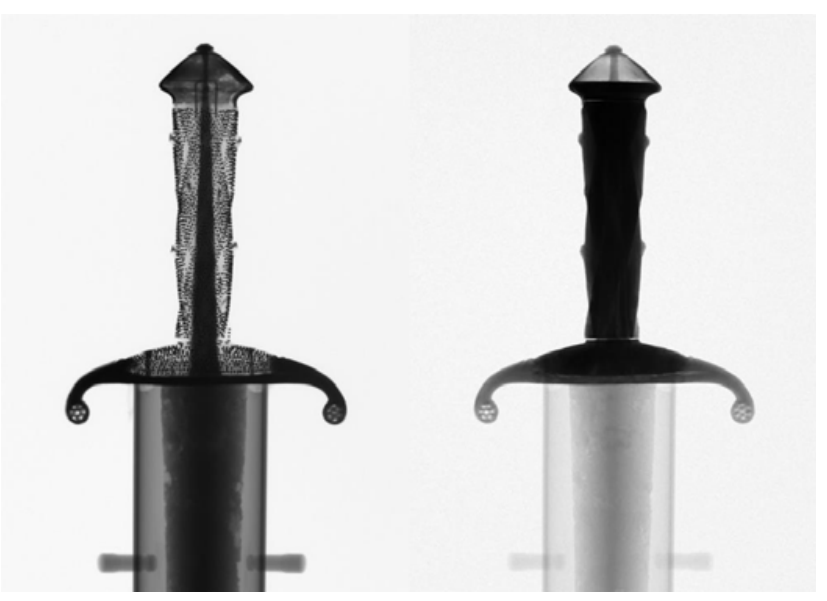

Figure 1. Non-invasive analysis of a historical sword from Switzerland [1] with $150 \mathrm{kV}$ X-rays (left) and thermal neutrons (right): although the inherent image quality is identical with respect to the pixel size, the contrast for the components (wood, steel, amalgam) is systematically different.

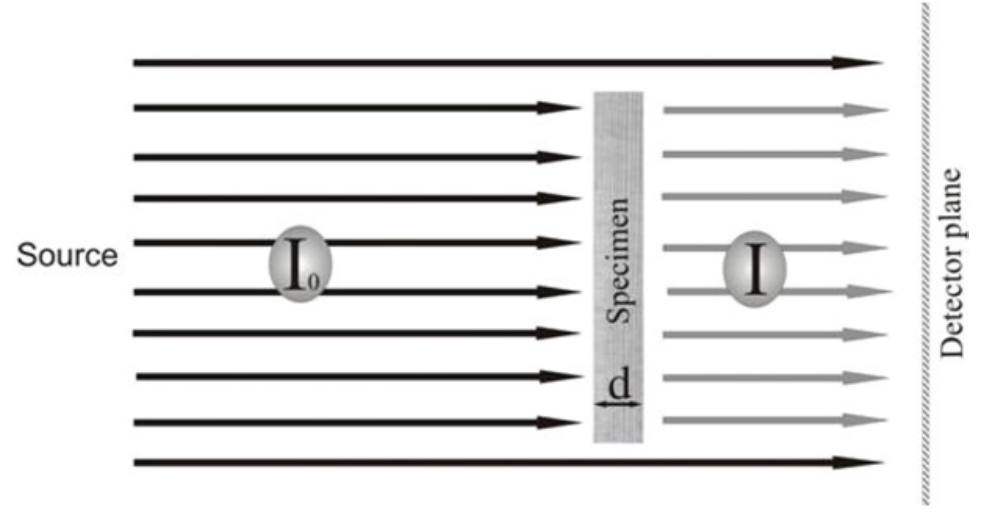

Figure 2. Simplified scheme of the neutron attenuation in a parallel beam by a sample with well-defined geometry perpendicular to the beam; the beam intensities $\mathrm{I}_{0}$ and $\mathrm{I}$ can be compared and information about the sample can be derived.

applications like polymer-electrolyte-membrane (PEM) fuel cells and Li-batteries and their behavior, hydrogen storage and it will end with some highlights of former nuclear fuel cladding investigations. Previously, the basics in neutron imaging, the facilities at PSI and the most common methods get introduced.

\section{The method of neutron imaging}

Figure 2 shows simplified how a flat plate sample with thickness d (in beam direction) reduces the beam intensity $\mathrm{I}_{0}$ and the detector behind can register only the intensity I. In this scheme, an ideal parallel beam is assumed what is hard to get in reality. Furthermore, the beam contains neutrons with a spectral distribution (e.g. a Maxwellian around $25 \mathrm{meV}$ ) while the attenuation of the sample is energy dependent (expressed by $\sigma(\mathrm{E})$ ). The detector has an energy response according to the absorption cross-section of the used converter material (Li-6, Gd, B-10, ...). 
Nevertheless, the attenuation of the sample can be described in a first-order approximation by BeerLambert's law:

$$
I=I_{0} \cdot e^{-\Sigma \cdot d}
$$

The attenuation coefficient $\Sigma=\mathrm{N} \cdot \sigma(\mathrm{E})$ is a material parameter defined by the neutron interaction probability (absorption and scattering) $\sigma$ and the material density $\mathrm{N}$.

$$
\mathrm{N}=\frac{\rho \cdot L}{M}
$$

With the Avogradro's constant L and the atomic number M it is easy to derive the material density $\rho$ if the sample thickness is known. On the other hand, if samples with a known homogenous composition but complicated inner structure are investigated it is possible to derive the material thickness $\mathrm{d}$ in beam direction instead.

For more complex systems, the inspection has to be performed from different viewing angles and the structure and composition can be calculated with mathematical tools in a reconstruction process. This technique of tomography is already established and common in X-ray labs and hospitals where the inverse of relation (1) is used to describe the investigated object as voxel matrix of attenuation coefficients $\Sigma(\mathrm{x}, \mathrm{y}, \mathrm{z})$. The same can be done with neutrons when the object is rotated around its vertical (or horizontal) axis in discrete angular steps. For the case of perfect parallel neutron beams, $180^{\circ}$ coverage is enough while conical beams require information from all $360^{\circ}$. Neutron tomography is now state-of-the-art in about 15 labs world-wide and can be applied in many research fields and for industrial investigations.

The experimental technique to obtain image data as "radiography $=2 \mathrm{D}$ " and "tomography $=3 \mathrm{D}$ " is based nowadays on digital imaging detectors, simplified shown as plane in Fig. 2. Most common are camera based systems which observe a neutron sensitive scintillators screen, mostly via a mirror to avoid radiation damage from direct exposure. There are also imaging plates, amorphous silicon flat panels and pixelated detectors in use with various pro and cons with respect to spatial and time resolution or the sensitivity and signal-to-noise characteristics.

Depending on the beam intensity, detector efficiency and required image quality, image data can be obtained within few milli-seconds and several minutes. This is orders of magnitudes better in efficiency than the outdated film technology provided in the past. However, there is still the need for improvement regarding higher spatial resolution and efficiency, in particular if the beam of a pulsed source has to be utilized. Although most of the neutron imaging facilities are situated at continuous sources (mostly reactor based), the new projects of neutron imaging facilities at pulsed spallation sources will require dedicated time-resolving systems in order to use the pulse structure for energy resolution in a time-offlight mode [2].

The example of a beam line for thermal neutrons is given in Fig. 3 for the NEUTRA station in sector 32 of the spallation neutron source SINQ [16] at PSI. It is surrounded by about $1 \mathrm{~m}$ concrete in all directions for shielding purposes. The beam conditions can be tuned according to the requirements with respect to collimation and intensity. Further conditions are chosen with respect to the neutron spectrum (filters, E-selectors) and the sample environment.

\section{Neutron imaging options}

The techniques to use neutrons in a transmission mode for the study of materials have been diversified in the recent years and new approaches and methods have been made available. Years ago, the field of "neutron radiography" was established as alternative and complementary tool for non-destructive testing, e.g. compared to X-ray radiography. 


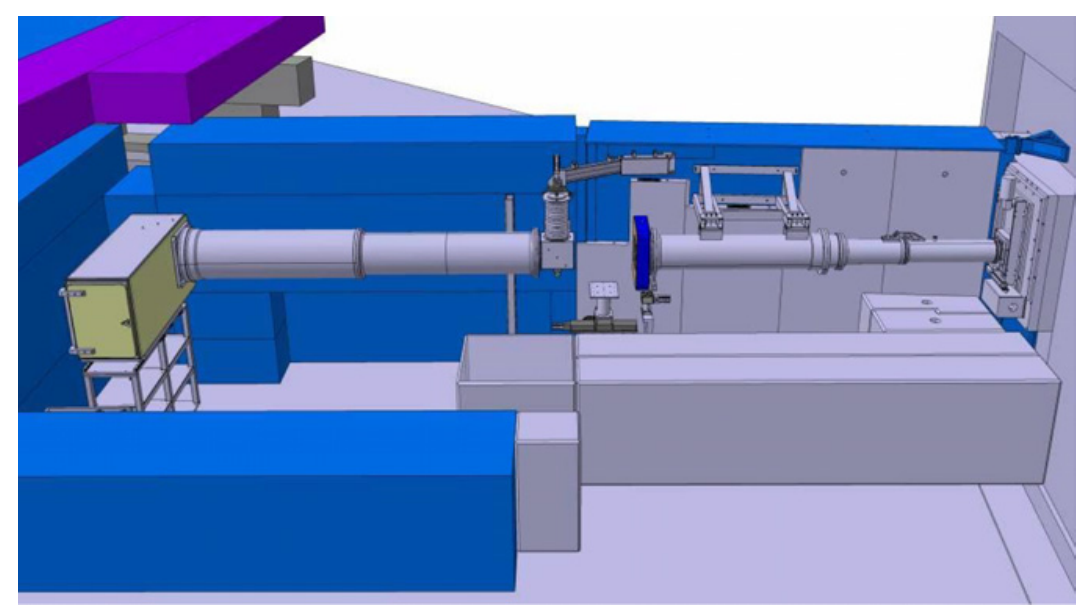

Figure 3. Overview drawing of the NEUTRA facility at the spallation neutron source SINQ of PSI: the beam comes from the right and two experimental positions can be used alternatively - the middle position with small field-of-view (FOV) but higher intensity and the end position with FOV of up to $40 \mathrm{~cm}$.

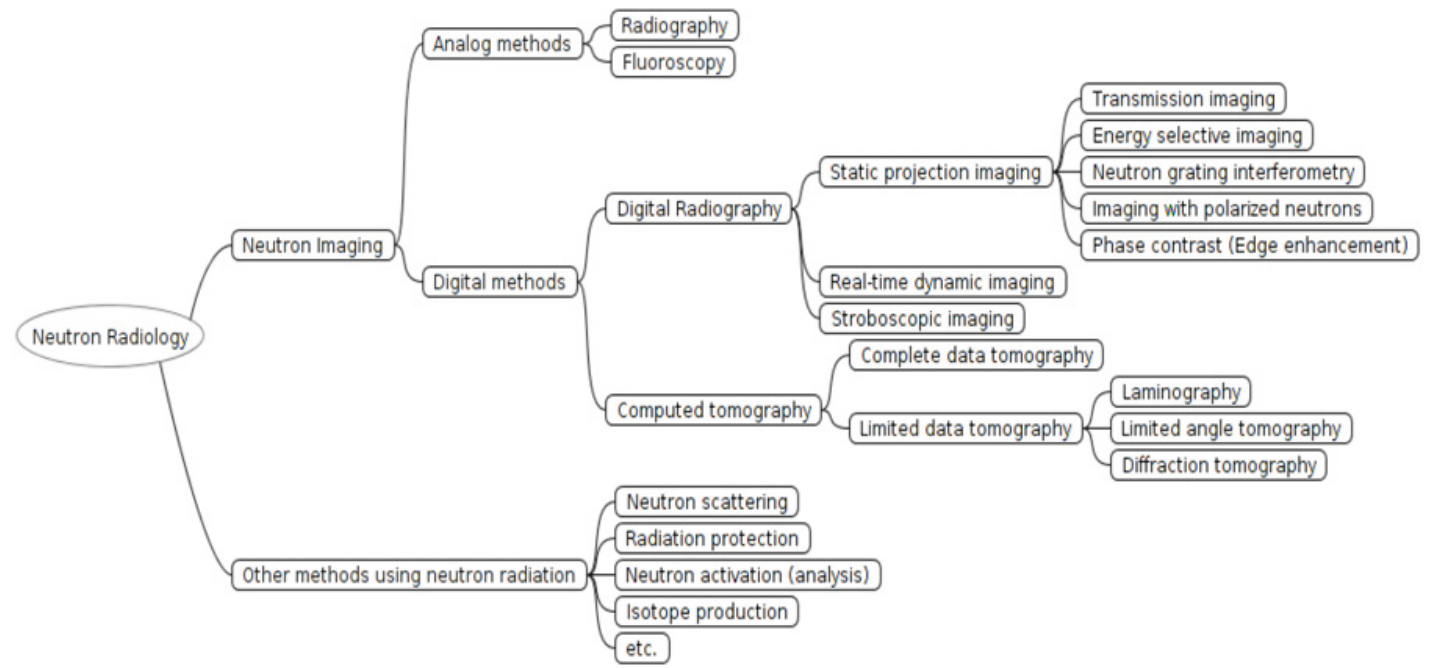

Figure 4. Preliminary scheme of options in the field of "neutron imaging" as the global category where the previously used "neutron radiography" plays a less important role. New methods are listed and sorted according to their inherent performance.

As shown in the overview scheme in Fig. 4, new trends do not fit into the category of "neutron radiography" and some new terms need to be introduced to describe the current situation. Since the discussion about that terminology is not yet completed, the scheme has to be taken as a draft only.

Driven by the developments in X-ray analysis and imaging, in particular by the strong synchrotron sources, there is a clear trend visible to increase the spatial resolution until the limits, which is presently less than $1 \mu \mathrm{m}$. This is hard to compete with neutrons for several reasons. Currently, standard imaging devices provide about $50 \mu \mathrm{m}$ resolution, a few systems comes down to $10 \mu \mathrm{m}$ [3]. It has to be taken into account higher resolution automatically results in smaller fields-of-view (FOV) in the observation of samples. In the framework of the project "neutron microscope" the NIAG group at PSI intends to become better than this present limit [4]. 


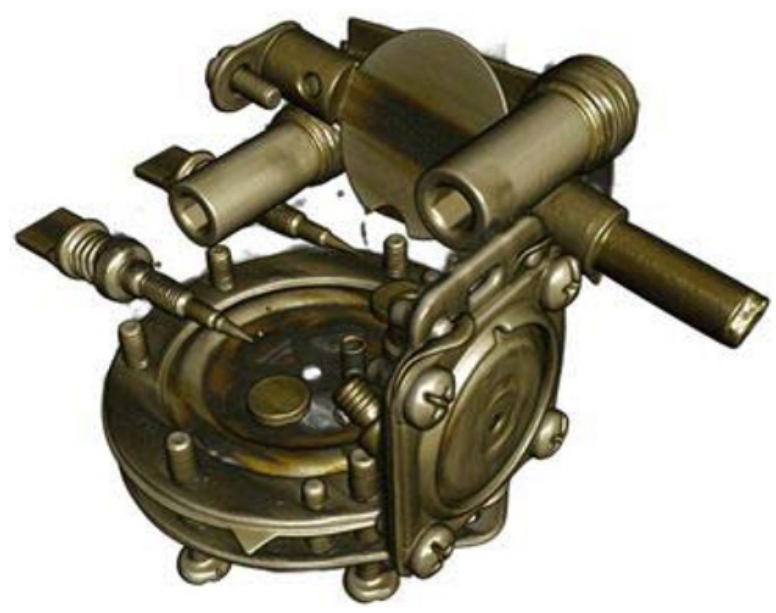

Figure 5. Visualization of neutron tomography data obtained from measurements of a car carburetor; the size of this sample is about $12 \mathrm{~cm}$. The data can be used for a target-performance comparison.

With respect to time resolution, the neutron source intensity is clearly a limiting factor. The neutron flux at the sample position can be up to about $10^{8} \mathrm{~cm}^{-2} \mathrm{~s}^{-1}$; assuming a pixel size of $0.1 \mathrm{~mm}$ we will get $10^{4}$ pixels per $\mathrm{cm}^{2}$ or 100 neutrons per pixel and s. Assuming $100 \%$ detection efficiency, a frame rate of 100 fps delivers about 1 detected neutron per pixel what might be the lower level for image acquisition. The best time resolution under these conditions will be than $1 \mathrm{~ms}$ exposure time.

Another approach is to study repetitive processes in stroboscopic manner by synchronizing a running process with the detection system. As shown in [5] for running combustion engines at $8000 \mathrm{rpm}$, the stacking of many individual frames at precisely the same position of the process delivers clear and noiseless individual images. Turning the process further, a full sequence of images can be obtained with good quality and the whole engine cycle can be visualized and measured.

Ten years ago the implementation of neutron tomography has been started. The perquisites were a stationary imaging detector (2D) with well-defined pixel size, a precise rotation table and computation power to perform the reconstruction work in reasonable time. In the meantime, neutron tomography became standard in several labs on different lengths scales (from 2 to $40 \mathrm{~cm}$ FOV and corresponding $1 / 2000$ in the resolution). The real sample sizes in the investigation are also defined by the attenuation in the beam, which depends on the material properties in the interaction with the neutrons. The related cross-section data for estimates according to (1) can be found e.g. in [6]. The samples should be transparent enough for all directions of observation during acquisition. An example for tomography data - which can directly compared to reality or technical drawings - is shown in Fig. 5.

A common setup at an imaging beam line provides the "white" spectrum from the initial source, which is either a thermal or a cold one with mean energies of either $1.8 \AA$ or $4 \AA$. There are several new aspects to narrow the energy band in neutron imaging: as the cross-sections of the observed materials are strongly energy dependent, a more precise quantification of the sample content via (1) becomes possible. On the other hand, scattering cross-sections of crystalline solids are caused by Bragg reflections at lattice planes. With well-defined neutron energies it becomes possible to visualize individual structures (textures) and to characterize materials in a direct and easy manner [7]. An example is given in Fig. 6. The selection of small energy bands can be done by mono-chromator devices based on turbines or single-crystal reflections [8]. In any case, the resulting beam has much less intensity and longer exposure time has to be taken into account. 

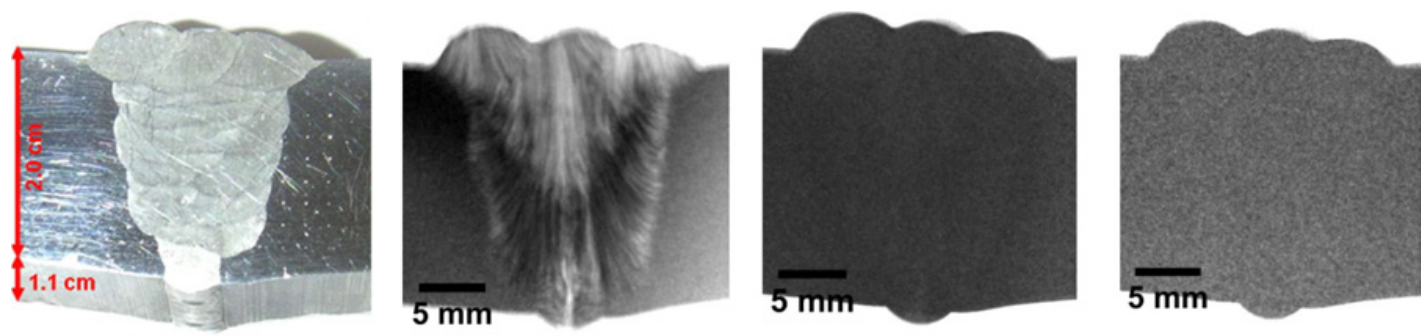

Figure 6. Energy-selective neutron imaging for the case of a stainless steel welt: photo (left), at $4.1 \AA$ (second left), at $5.1 \AA$ and with the full spectrum (right).

When the energy band can be reduced further, e.g. in time-of-flight mode, even the internal strain in solids become visualized [9]. This is the potential for future installations at pulsed spallation sources.

For completeness, we mention here the imaging with polarized neutrons [10] with the potential of the visualization for magnetic fields and structures. Further, the neutron grating interferometry [11] has a high potential for magnetic domain wall measurements and the searching for small-angle phenomena in direct mode on the macro-scale. Phase contrast imaging and refractive edge effects do complete the palette of new methodical approaches which will trigger new application fields in the future.

\section{Comparison to X-ray studies}

Historically, X-rays were found about 50 years earlier than free neutrons became available. In the meantime, the time difference with respect to imaging methods of neutrons and X-rays, respectively, has been narrowed. However, neutron imaging is still limited in some respect, e.g. intensity and resolution, by physical reasons.

Both imaging techniques are similar and complement each other well with advantages and drawbacks, individually different for each individual material.

The interaction of X-rays is mainly with the electrons in the atomic shell, while neutrons deal mainly with the atomic nuclei. This gives the clear contrast differences in the transmission imaging: for $\mathrm{X}$-rays the rule holds: the higher the atomic mass, the higher the contrast. This is not at all valid for the neutrons, where already some light elements $(\mathrm{H}, \mathrm{Li}, \mathrm{B})$ interacts strongly while some heavy elements are transparent $(\mathrm{Pb}, \mathrm{Bi}, \mathrm{U}, \mathrm{Au})$.

This explains the difference in Fig. 1: the wooden handle with much hydrogen content is very dark in the neutron image while transparent with X-ray. At the same time, the metal zones deliver high contrast for the X-rays, but less contrast for neutrons.

\section{Facilities at PSI (and world-wide)}

The International Society for Neutron Radiology (ISNR) [12] and the Research Reactor Section of IAEA [13] organized a survey about existing and planned facilities for neutron imaging in a world-wide context. Based on a questionnaire, there is now a data base available for about 50 installations with differing performance. In order to compare these individually built systems a categorization into the following branches can be done:

- User facilities: state-of-the-art installations with digital detection systems, invitation for external user, beam time allocation according to a proposal scheme;

- In-house usage: the facility might have high performance, but the access from external users is not (yet) organized and foreseen; 


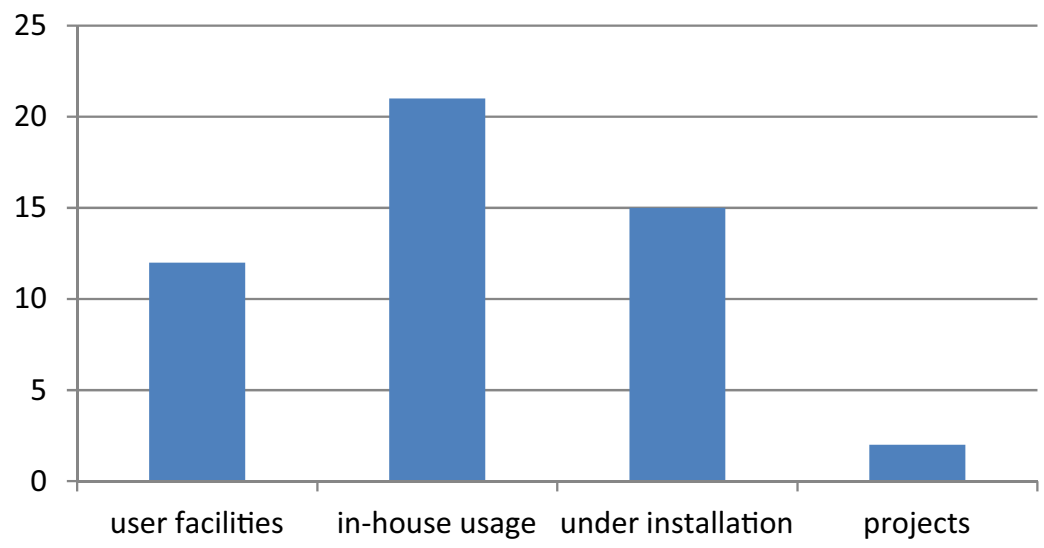

Figure 7. Survey of neutron imaging facilities world-wide with respect of their usage and installation status [12].

- Under installation: the system is not yet completed;

- Projects: a facility is planned, but the installation has not yet started.

Among the "user facilities" we have two stations running at PSI: NEUTRA at the thermal beam port [14] and ICON at a cold one [15]. Both are located at the Swiss spallation neutron source SINQ [16] which is successfully under operation since 1997.

Both facilities are equipped with modern neutron imaging systems covering a wide range of observation fields and related pixel size. In this manner, the full beam size of $40 \mathrm{~cm}$ in diameter can be used for large objects. For small samples, the demand for highest possible resolution is now covered with the micro-tomo setup [3], which will be improved further by an ultimate device with about 2-5 $\mu \mathrm{m}$ pixel size [4]. Given by the beam intensity, images can be obtained within a few seconds using the full range of the detection system.

ICON is equipped with devices to reduce the spectral range of the neutrons in order to enable "energy selective" neutron imaging. The resolution can be varied between 2 and $15 \%$ of the full spectrum. Furthermore, a neutron grating interferometer can be installed [11] in order to investigate "phase contrast" or "dark field" phenomena like magnetic domain structures in bulk metallic samples.

NEUTRA, in addition to the well collimated and homogeneously illuminated thermal neutron beam, can provide a setup for X-ray imaging (XTRA options) with pixel-wise referencing for data fusion of neutron and X-ray data. The high intensity tube can be operated up to $320 \mathrm{kV}$.

\section{Application of neutron imaging (overview)}

Neutron Imaging has developed towards a universal research tool for many users from different branches of science. Due to the flexibility in the FOV and resolution, the setup can be adapted to the requests of the research task, depending on the needed contrast and the required sample transmission. A very common approach, used in several modifications is the study of porous media with respect to the structure and void partitions, but also for the uptake of water and other organic liquids. Other approaches deals with metals, their structure, modification and transformation, including joining and destruction (e.g. by corrosion).

The link to industrial applications is well established since neutron imaging complements the common X-ray techniques. Here, the higher transmission trough metals and the high contrast for organic materials are favorably used. Some promising approaches you will find below. 


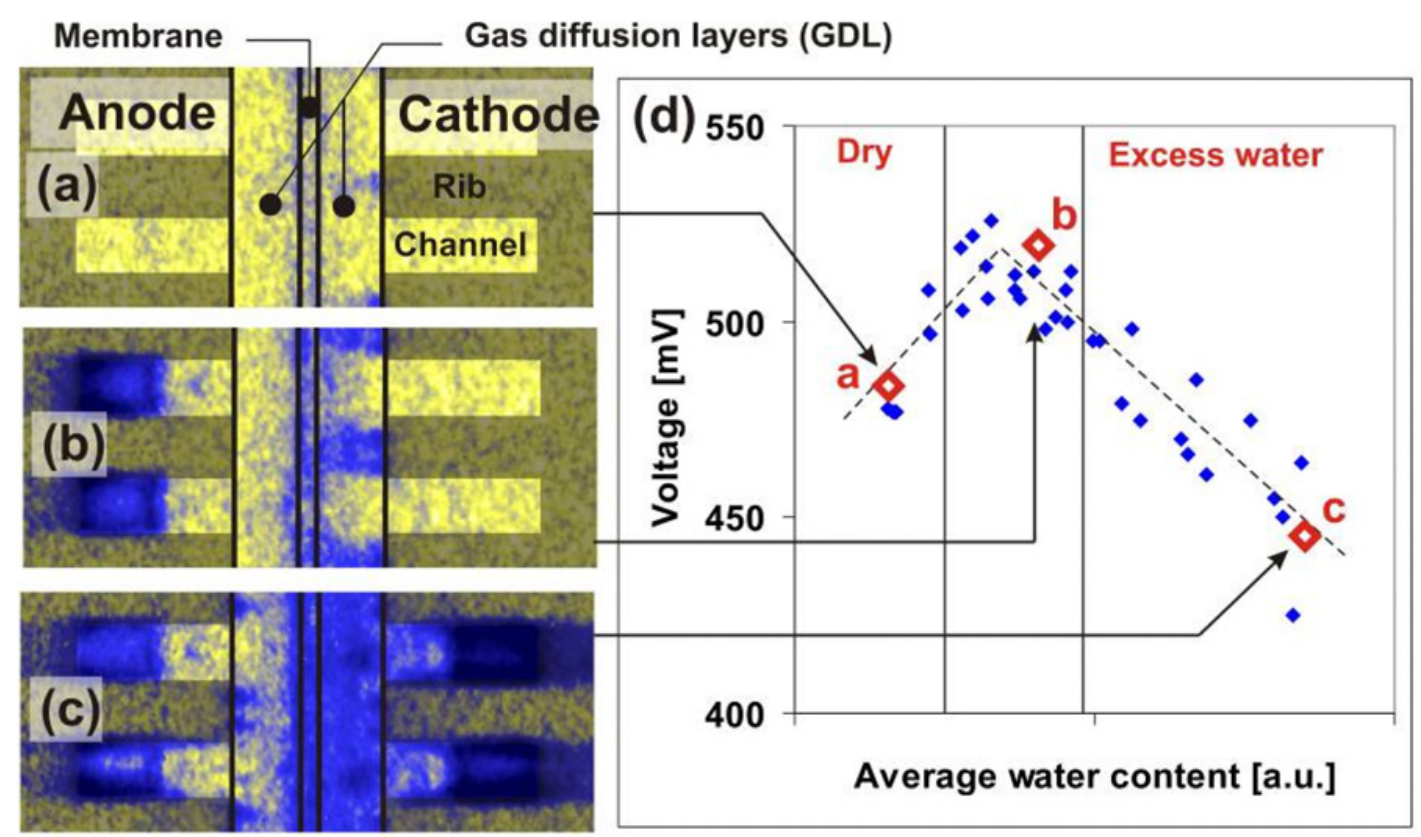

Figure 8. Water distribution in a test PEM fuel cell next to the membrane region: water distribution (left), comparison of the accumulated water amount with the voltage within the cell.

\section{Applications in the energy field}

\section{Polymer-Electrolyte Membrane (PEM) - Electric fuel cells}

The combination of hydrogen and oxygen gas in an electro-chemical process within a interaction membrane delivers the separation of charge $\rightarrow$ creation of voltage and generates reaction water on the other hand. Certain amount of water is always needed to keep the fuel cell well running and stimulates the catalytic reaction, but too much water will disturb the gas flow and the overall performance will go down.

In-situ studies with operational PEM fuel cell are performed at our beam lines with success, where the water generation, accumulation and distribution can be observed in high spatial and temporal resolution in comparison to the electro-chemical parameters, derived on-line.

An example of such a study is given in Fig. 8. We relate the observation of water production within the cell with the electrical performance, given by the measured voltage. The water amount can directly be quantified from the digital neutron imaging data since the cross-section data for water under our spectral conditions are well-known. It can be observed that highest voltage is reached when a certain level of moisture is in the membrane region. Less or too much water will reduce the performance of the fuel cell. Therefore, the water management in the fuel cells is very important for an efficient operational regime. However, these conditions are different for each assembly and influenced by different conditions (cell temperature, moisture inlet, gas composition, membrane structure, ...). Fuel cell studies by means of neutron imaging methods are very important for the understanding and the tuning of model and real fuel cell systems. They are performed either for special model assemblies in order to test specific parameters or conditions or with near commercial systems on full scale for verification of the specifications. The observation field can therefore differ between $3 \mathrm{~cm}$ and $40 \mathrm{~cm}$, fitting to the beam and detector dimensions. 

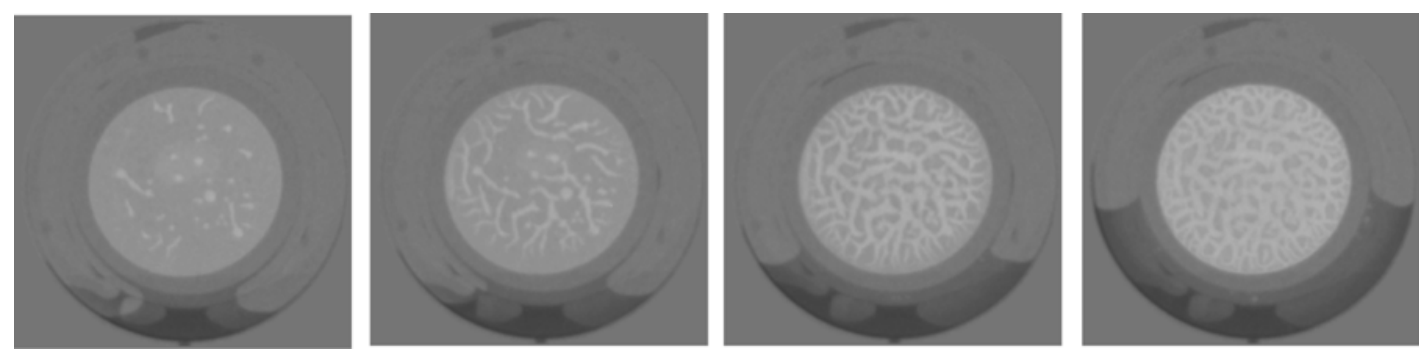

Figure 9. Evolution of the gas production within the electrolyte of a Li-ion battery assembly during the increase of induced current density. This test cell was fully operational (diameter $4 \mathrm{~cm}$ ). Data taken from [18].

Recently, we studied the behavior of fuel cells under low temperature conditions where the risk for ice generation is high. Since ice will disturb or hinder the electro-chemical process, it should be known at which temperature and under which other conditions the water $\rightarrow$ ice transfer starts. By means of neutrons with different energies, the ratio of the attenuation parameters is a good indicator for these transitions [17].

\section{Li-ion batteries}

Due to the increased usage of mobile electronics and electric devices and for the short term storage of electrical energy in general, batteries attracted very high attention. The improvement of the storage capacity, their durability and power density requires the full understanding of the electro-chemical conditions under the influence of many parameters.

By means of neutron imaging investigations we are able to study the in-situ process of gas formation in thin model battery assemblies (Fig. 9) in an online sequence with a time resolution of one frame within a few seconds while the derived current flow from the battery is increase. This becomes possible since the contrast for Li is high with respect to the transmission of thermal neutrons compared to the gas portion. These data can be used directly to quantify the amount of the produced gas amount in relation of the cell parameters.

Further studies with Li-ion batteries are concentrated on the direct observation of the Li diffusion within the individual layers on a micro-structural level using high resolution detectors. For this purpose, it might become possible to use the enormous contrast difference between the two Li isotopes Li-6 and Li-7. The usage of depleted in Li-6 agents and a high resolution detector enables to study the migration process during charge and discharge in spatial and temporal resolution. Such studies are under preparation yet.

\section{Hydrogen storage}

The high attenuation of thermal neutrons by hydrogen enables to study and to quantify small amounts of hydrogen in the metallic matrix of the reservoir. As storage assemblies are either under high pressure or related to high temperature gradients, the structures are sealed with steel of remarkable thickness. Even then, neutrons can penetrate and have reasonably high sensitivity to investigate the performance of the storage device. As shown in Fig. 10, such studies can either be performed in a tomography experiment, where the distribution is fixed over the observation time of a few hours or in real-time investigations in the radiography (2-dimensional) mode. A quantification of the amount of stored hydrogen can be performed in both cases in good precision. 


\section{Hydrogen Storage}

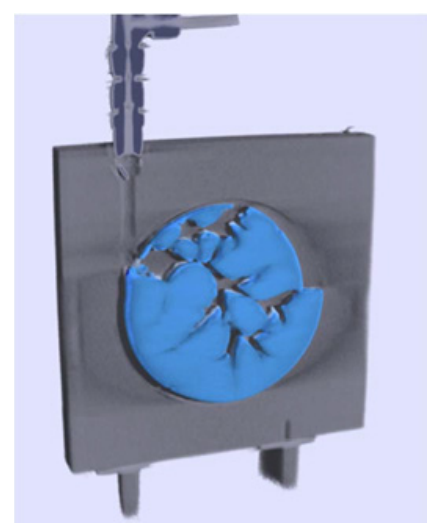

Figure 10. Tomographic view onto the stored amount of hydrogen in the metallic matrix. Data are taken from [19].

\section{Nuclear fuel inspection}

By means of neutron transmission measurements it is possible to investigate nuclear fuel since the attenuation by uranium is more moderate than it is for X-rays $\left(\mu(100 \mathrm{kV})=40 \mathrm{~cm}^{-1}\right)$. Furthermore, it is possible to distinguish the two relevant isotopes U-235 and -238 very effectively because their attenuation is quite different. In this manner it becomes possible to measure the degree of enrichment non-invasively with good precision since it linearly related to the effective attenuation cross-section. The contrast variations in Fig. 11 are the indication of the difference in the enrichment in the fuel pellets.

More complicated is the study of spent nuclear fuel. Because it is highly radioactive the high gamma background hinders the usage of radiation sensitive in direct view. Therefore, indirect techniques have to be applied as described in [20]. In such cases, the material damage from the fission process and the distribution of fission products can be investigated.

Of similar importance is the study of the fuel cladding, mainly produced from $\mathrm{Zr}$ alloys. During normal operation, but also accidental conditions an increase of the hydrogen ingress into the material can happen. It is of high relevance to understand this behavior because the hydrogen of high amount causes embrittlement in the cladding material and destruction might happen as shown in Fig. 10. The dark areas of this radiography image correspond to higher $\mathrm{H}$ amounts which are quantified in the diagram below.

\section{Further trends in neutron imaging}

Neutron imaging techniques have gained more attraction and acceptance in the past years what is visible in different new installations world-wide. Since the newest reactor sources are situated in developing countries, their support for building suitable beam lines is essentially. This can be done either by consultancy or financial support, e.g. from IAEA.

The methods of neutron imaging are under further development and improvement. Following the demands of the facilities' users, the increase in the spatial resolution is an important issue. For this purpose, dedicated detection systems are under development $[4,21]$.

Although the intensity of the neutron sources can hardly be raised by orders of magnitude, time dependent studies become possible with frame rates of several ten per second. With modern CMOS sensors, the readout delay (usual with CCDs) has become no issue. Stroboscopic investigations have been already performed for engines on $8000 \mathrm{rpm}$, more is technically possible. 


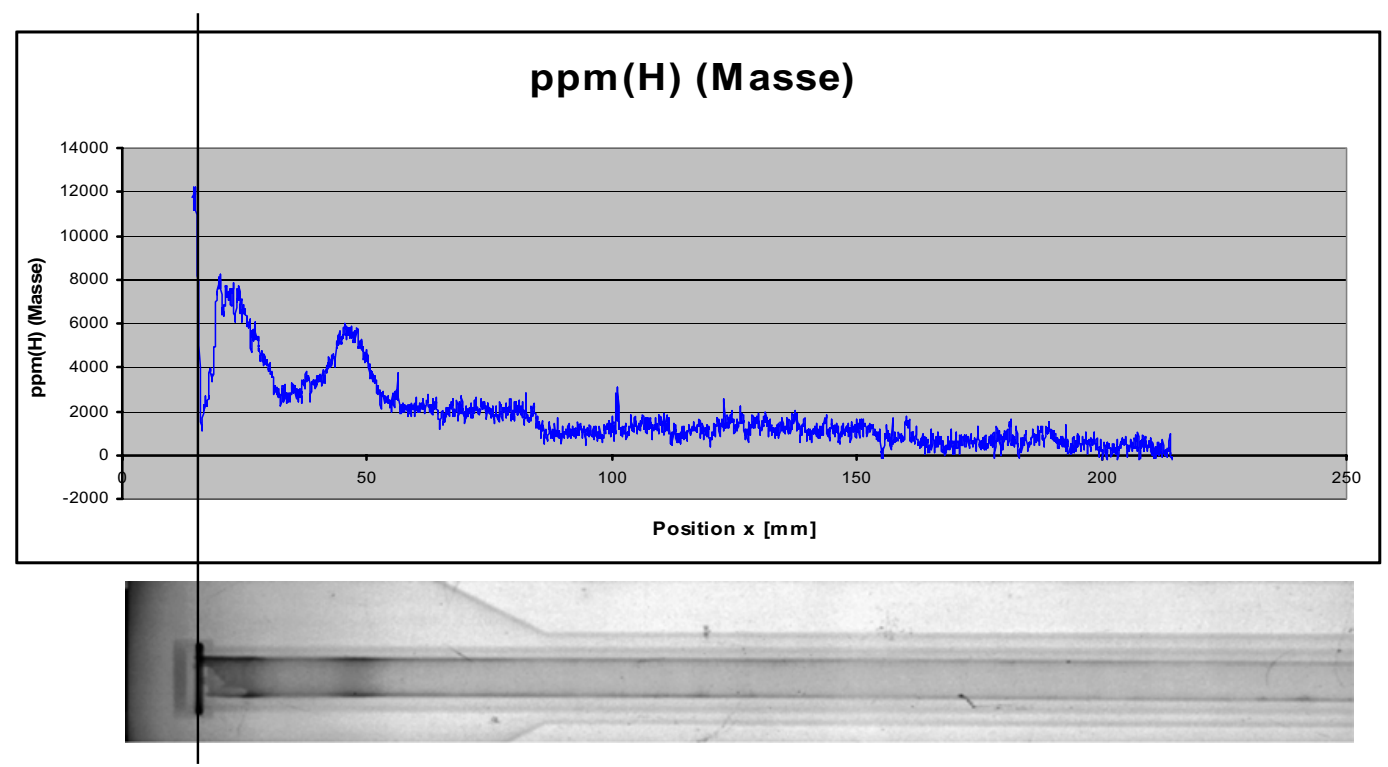

Figure 11. Qualitative and quantitative investigation of the hydrogen ingress in the cladding of a fuel rod after long-term operation. The fuel pellets were withdrawn from the assembly for this study.
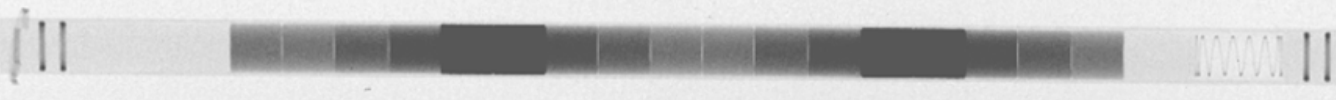

Figure 12. Transmission image of fuel rod with pellets of different enrichment, what results in the attenuation contrast variation. The very dark regions correspond to fuel with a "poisoning" with Gd for the burnup control.

The improvement in the energy selective neutron imaging for studies near Bragg edges for the visualization of crystalline structures and the observation of stress-strain phenomena will be optimizes as soon as time-of-flight systems are available at the beam lines of pulsed spallation sources. Several are now under completion.

The usage of the spin-state orientation of the neutron enables the access to the study of magnetic phenomena inside and closed to bulk magnetic assemblies under various conditions (temperature, pressure, different external fields. There are already some facilities for polarized neutron imaging, but an increase in number is needed in order to establish "polarized neutron imaging" towards a routine method, also for industrial purposes.

\section{Conclusions \& outlook}

Neutron imaging is established at prominent neutron sources as a valuable method for research with the high potential for many practical applications. Compared to the more common X-ray techniques, the performance in neutron imaging is mostly equivalent and several inherent advantages can be used favorably. For the improvement of the methodology, the access to more beam ports with suitable layout is essential. In this way, very useful conditions can be offered for the scientific user community and to industrial partners. 


\section{References}

[1] D. Berger, K. Hunger, S. Bolliger-Schreyer, D. Grolimund, S. Hartmann, J. Hovind, F. Mueller, E.H. Lehmann, P. Vontobel, M. Woerle, New insights into the manufacturing process and decoration technique of the axe from Thun-Renzenbuehl, The Antiquaries Journal 93, 1 (2013).

[2] E. H. Lehmann, NEUWAVE-6: Progress and Perspectives in Neutron Imaging, Neutron News August 2014, doi: 10.1080/10448632.2014.930617.

[3] E. H. Lehmann, G. Frei, G. Kuehne, P. Boillat. The micro-setup for neutron imaging: A major step forward to improve the spatial resolution Nuclear Instruments \& Methods in Physics Research Section a-Accelerators Spectrometers Detectors and Associated Equipment 576 (2-3): 389-396 Jun 212007.

[4] P. Trik, E. H. Lehmann, Extending the spatial resolution of neutron imaging facilities at PSI - the Neutron Microscope project, to be published in Physics Proceedings.

[5] Gruenzweig, C., "Visualization of a Fired Two-Stroke Chain Saw Engine Running at Idle Speed by Dynamic Neutron Radiography," SAE Technical Paper 2010-32-0013, 2010, doi: 10.4271/2010-32-0013.

[6] https://www-nds.iaea.org/exfor/endf.htm.

[7] E.H. Lehmann, S. Peetermans, L. Josic, H. Leber, H. van Swygenhoven, Energy-selective neutron imaging with high spatial resolution and its impact on the study of crystalline-structured materials, Nuclear Instruments \& Methods in Physics Research Section A-Accelerators Spectrometers Detectors and Associated Equipment 735, 102-109.

[8] S. Peetermans and E. H. Lehmann. Simultaneous neutron transmission and diffraction contrast tomography as a non-destructive 3D method for bulk single crystal quality investigations. Journal of Applied Physics 114:124905, 2013.

[9] R. Woracek et al., Neutron Bragg-edge-imaging for strain mapping under in situ tensile loading, J. Appl. Phys. 109, 093506 (2011); http: //dx.doi.org/10.1063/1.3582138.

[10] N. Kardjilov, I. Manke, M. Strobl, A. Hilger, W. Treimer, M. Meissner, T. Krist, J. Banhart, Nat. Phys. 4 (2008), p. 399 doi: 10.1038/nphys912.

[11] F. Pfeiffer, C. Gruenzweig, O. Bunk, C. David et al., Phys. Rev. Lett. 96, 215505 (2006).

[12] www.isnr.de/.

[13] http://nucleus.iaea.org/RRDB/RR/ReactorSearch.aspx?filter=0.

[14] http://www.psi.ch/niag/niag.

[15] A. P. Kaestner, S. Hartmann, G. Kühne, G. Frei, C. Grünzweig, L. Josic, F. Schmid, and E. H. Lehmann. The ICON beamline - a facility for cold neutron imaging at Sinq. Nuclear Instruments \& Methods in Physics Research Section A-Accelerators Spectrometers Detectors and Associated Equipment, 659:387-393, 2011.

[16] www.psi.ch/sinq/.

[17] Biesdorf J., Oberholzer P., Bernauer F., Kaestner A., Vontobel P., Lehmann E. H., Schmidt T. J., Boillat P., Dual Spectrum Neutron Radiography: Identification of Phase Transitions between Frozen and Liquid Water Physical Review Letters 112, 248301 (2014). doi: 10.1103/PhysRevLett.112.248301.

[18] D. Goers, M. Holzapfel, W. Scheifele, E. Lehmann, P. Vontobel, and P. Novák, In situ neutron radiography of lithium-ion batteries: The gas evolution on graphite electrodes during the charging. Journal of Power Sources 130 (2004) 221-226, doi: 10.1016/j.jpowsour.2003.11.065.

[19] P.K. Pranzas et al.; Advanced Engineering Materials 13 (2011), 730-736.

[20] M. Tamaki, K. Iida, N. Mori, E. H. Lehmann, P. Vontobel and M. Estermann, Dy-IP characterization and its application for experimental neutron radiography tests under realistic 


\section{JDN 21}

conditions, Nuclear Instruments \& Methods in Physics Research Section A-Accelerators Spectrometers Detectors and Associated Equipment 542 (1-3): 320-323 Apr 212005.

[21] A. S. Tremsin, "High resolution microchannel plates neutron counting detectors and their applications in neutron radiography, diffraction and resonance absorption imaging", Neutron News 23 (2012) 35. http://dx . doi .org/10.1080/10448632.2012.725341. 